\title{
Convergence to Equilibrium in Degenerate Parabolic Equations with Delay
}

\author{
R. Laister \\ Department of Engineering Design and Mathematics, University of the West of England, \\ Frenchay Campus, Bristol BS16 1QY, England
}

\begin{abstract}
In [10], Busenberg \& Huang (1996) showed that small positive equilibria can undergo supercritical Hopf bifurcation in a delay-logistic reaction-diffusion equation with Dirichlet boundary conditions. Consequently, stable spatially inhomogeneous time-periodic solutions exist. Previously in [5] Badii, Diaz \& Tesei (1987) considered a similar logistic-type delay-diffusion equation, but differing in two important respects: firstly by the inclusion of nonlinear degenerate diffusion of so-called porous medium type, and secondly by the inclusion of an additional 'dominating instantaneous negative feedback' (where terms local in time majorize the delay terms, in some sense). Sufficient conditions were given ensuring convergence of non-negative solutions to a unique positive equilibrium.

A natural question to ask, and one which motivated the present work, is: can one still ensure convergence to equilibrium in delay-logistic diffusion equations in the presence of nonlinear degenerate diffusion, but in the absence of dominating instantaneous negative feedback? The present paper considers
\end{abstract}

Email address: Robert.Laister@uwe.ac.uk (R. Laister) 
this question and provides sufficient conditions to answer in the affirmative. In fact the results are much stronger, establishing global convergence for a much wider class of problems which generalize the porous medium diffusion and delay-logistic terms to larger classes of nonlinearities. Furthermore the results obtained are independent of the size of the delay.

Keywords: Delay, degenerate, parabolic, diffusion, logistic, convergence

\section{Introduction}

Let $\Omega$ be a bounded domain in $\mathbb{R}^{n}$ with boundary of class $C^{2+\alpha}$ for some $\alpha \in(0,1)$. Define $Q_{T}=\Omega \times(0, T], S_{T}=\partial \Omega \times(0, T]$ and $\Gamma_{r}=\bar{\Omega} \times[-r, 0]$. We consider the following nonlinear degenerate diffusion equation with delay

$$
(D)\left\{\begin{aligned}
\partial_{t} u=\Delta \phi(u)+f(u(x, t)) h(u(x, t-r)) & \text { in } Q_{T}, \\
u=0 & \text { in } S_{T}, \\
u=\eta_{s} \geq 0 & \text { in } \Gamma_{r},
\end{aligned}\right.
$$

where $r>0$ is the delay and $\eta_{s}(x):=\eta(x, s)$ the initial data. As usual $\Delta$ denotes the Laplacian operator and $\partial_{t} u$ denotes the partial time derivative $\partial u / \partial t$. Throughout we will write $Q$ instead of $Q_{\infty}$ and we will sometimes abuse notation slightly by writing $u(t)$ instead of $u(\cdot, t)$, for a function $u(x, t)$.

The associated time-independent stationary problem for (D) is given by

$$
(D S) \quad\left\{\begin{aligned}
\Delta \phi(u)+f(u) h(u)=0 & \text { in } \Omega \\
u=0 & \text { in } \partial \Omega .
\end{aligned}\right.
$$

This paper is concerned with the large-time behaviour of non-negative solutions of (D) and their convergence to solutions of (DS) as $t \rightarrow \infty$. Its novelty lies in the combination of three distinct types of nonlinearity: degenerate 
diffusion $\phi(u)$, local source term $f(u)$ and time delay $h(u(x, t-r))$. Such problems are often considered as models in population dynamics where $\phi(u)$ represents movement of individuals to avoid over-crowding, $f(u)$ an intrinsic growth rate and $h(u(x, t-r))$ a delayed response due to gestation periods, resource conversion, incubation periods, etc.

There is a large literature relating to problem (D) when delays are absent $(r=0)$, see $[3,23,27]$ for an overview and extensive bibliographies. We will make use of several key results from this literature on degenerate parabolic equations, using it mainly to provide suitable comparison solutions for the solutions of (D). Several authors have considered the non-delay degenerate parabolic case in the presence of periodic forcing terms and established existence and attractivity properties of periodic solutions [14, 26]. There are also many works dealing with the case of linear diffusion and nonlinear delay terms $(r>0)$, see $[19,24,29]$ for an overview and references. More recently researchers have considered problems incorporating degenerate diffusion, delay and periodic forcing $[28,30]$.

In [10] the authors considered the following linear diffusion case $\phi(u)=u$ with logistic delay $h(u)=1-u$ and local source term $f(u)=k u(k>0)$ :

$$
\begin{array}{r}
\partial_{t} u=u_{x x}+k u(x, t)(1-u(x, t-r)), \quad x \in(0, \pi), \quad t>0, \\
u=0, \quad x=0, \pi, \quad t>0 .
\end{array}
$$

It is well known that (1-2) possesses a unique positive equilibrium $U_{k}$ for all $k>1$, and only the trivial equilibrium $U=0$ when $k<1$, with $\left\|U_{k}\right\|_{\infty} \rightarrow 0$ as $k \rightarrow 1^{+}$. In the case of no delay $(r=0)$ it is also well known that $U_{k}$ attracts all non-negative non-trivial solutions for $k>1$; when $k<1$ the trivial equilibrium $U=0$ attracts such solutions. In [10] the authors fixed $k$ slightly 
greater than, sufficiently close to, 1 and showed that $U_{k}$ undergoes Hopf bifurcation as $r$ increases through an infinite sequence of positive values $0<$ $r_{0}(k)<r_{1}(k)<r_{2}(k), \ldots$ In particular they showed that the first bifurcation at $r_{0}(k)$ is supercritical, giving rise to stable, spatially inhomogeneous, timeperiodic solutions of (1-2). Consequently, there exist values of $k$ and of the delay parameter $r$ for which the (small) positive equilibrium $U_{k}$ is not locally attractive. The present work was motivated in part by asking whether this kind of 'delay-induced instability' can occur when linear diffusion is replaced by nonlinear degenerate diffusion, such as $\Delta\left(u^{m}\right)$ for $m>1$ (the so-called porous medium slow diffusion operator).

No comprehensive literature exists for degenerate parabolic equations including delay terms (and without periodic forcing). To the best of the author's knowledge the only paper in a similar spirit to the present one is [5]. There the authors considered the equation

$$
\partial_{t} u=\Delta u^{m}+u\left(a(x)-b(x) u-\int_{-\infty}^{t} u(x, s) K(x, t-s) d s\right),
$$

where $b$ and $k$ are non-negative functions and the positivity set of $a$ in $\Omega$ is non-empty. Crucially, and in contrast to the present paper where $b \equiv 0$, it was assumed in [5] that $b>0$ on $\bar{\Omega}$. Furthermore, in order to guarantee convergence to a unique positive equilibrium, $b$ was assumed [5, Theorem $2.5]$ to satisfy the stronger condition

$$
b(x) \geq \int_{0}^{\infty} K(x, s) d s, \quad \forall x \in \Omega .
$$

Assumptions such (4) are sometimes referred to as 'diagonally dominant' or having 'negative instantaneous feedback' in the delay-differential equation 
literature and in the theory of competitive population dynamics. Mathematically this property is often used to overcome the absence of a comparison principle in situations where the delay term has a negative response effect, corresponding to $h^{\prime}<0$ in our context. See $[16,15,17,19]$.

Such terminology refers to the assumption that the local, instantaneous term $b u$ dominates the non-local, delayed term $K \star u$ (the convolution term in $(3))$. The work of [5] provides a second motivation for the present paper, namely to obtain sufficient conditions for global convergence of non-negative solutions in the absence of negative instantaneous feedback (i.e. with $b=0$.)

The remainder of the paper is structured as follows. In Section 2 we define the solution concepts for the problems encountered and establish preliminary existence-uniqueness results. In Section 3 we summarize and extend some known results from the literature concerning sign-indefinite degenerate parabolic equations. Section 4 contains the main results of the paper. Sufficient conditions will be given which ensure global convergence of nonnegative solutions of $(\mathrm{D})$ to a positive equilibrium, see Theorem 4.1. The class of problems for which the results are applicable include the logistictype reaction term described above as a special case. The final Section 5 contains some examples and discussion.

\section{Global existence and uniqueness for the delay problem}

Let $\mathbb{R}^{+}=[0, \infty)$. We begin with the following assumptions:

(A1) $\phi \in C^{1}\left(\mathbb{R}^{+}\right), \phi(0)=\phi^{\prime}(0)=0, \phi^{\prime}>0$ on $(0, \infty), \phi^{-1}$ exists and $\phi^{-1} \in C^{\alpha}\left(\mathbb{R}^{+}\right)$, there exist $\gamma, \delta>0$ such that $\phi$ is convex on $(0, \delta)$ and $u \phi^{\prime}(u)<\gamma \phi(u)$ on $(0, \infty)$. 
(A2) $f \in C^{1}\left(\mathbb{R}^{+}\right)$and $f(0)=0$.

(A3) $\lim _{u \rightarrow \infty} f(u) / \phi(u)=0$.

From here onwards $g: \mathbb{R}^{+} \rightarrow \mathbb{R}^{+}$will denote the function given by

$$
g(u)=f\left(\phi^{-1}(u)\right) .
$$

We observe that $g$ has the following properties: $g \in C^{1}(0, \infty) \cap C^{\alpha}\left(\mathbb{R}^{+}\right)$, $g(0)=0$ and $g(u) / u \rightarrow 0$ as $u \rightarrow \infty$ (sublinearity at infinity).

Let us introduce the inhomogeneous, degenerate parabolic problem

$$
\text { (П) }\left\{\begin{aligned}
\partial_{t} u=\Delta \phi(u)+H(x, t) f(u) & \text { in } Q_{T}, \\
u=0 & \text { in } S_{T}, \\
u=\eta_{0} \geq 0 & \text { in } \Omega,
\end{aligned}\right.
$$

where $H \in C(\bar{Q})$ and $\eta_{0} \in C(\bar{\Omega})$. Such problems appear as a special case of those studied in [2].

\section{Definition 2.1.}

(a) (i) A non-negative weak solution u of problem (II) is a function $u \in C\left([0, T], L^{1}(\Omega)\right) \cap L^{\infty}\left(Q_{T}\right)$ satisfying

$$
\begin{aligned}
\int_{\Omega} u(\tau) \zeta(\tau)-\eta_{0} \zeta(0) d x & -\int_{Q_{\tau}} u \partial_{t} \zeta+\phi(u) \Delta \zeta d x d t \\
& =\int_{Q_{\tau}} H(x, t) f(u) \zeta d x d t
\end{aligned}
$$

for all $\tau \in[0, T]$ and $\zeta \in C^{2}\left(\bar{Q}_{T}\right)$ with $\zeta \geq 0$ and $\zeta=0$ on $S_{T}$. $A$ weak subsolution $v$ (respectively supersolution $w$ ) of problem (П) is defined similarly, but with equality replaced by $\leq$ (respectively $\geq)$ in (6). 
(ii) A non-negative classical solution u of problem (П) is a function $u \in C\left(\overline{Q_{T}}\right)$ for which the partial derivatives $\partial_{t} u$ and $\Delta \phi(u)$ exist and are continuous in $Q_{T}$ and satisfies (П) pointwise. A classical subsolution $v$ (respectively supersolution $w$ ) of problem (П) is defined similarly but with equality replaced by $\leq$ (respectively $\geq$ ) in (II).

(b) Non-negative weak and classical solutions of problem (D) are similarly defined, with $H(x, t)$ replaced by $h(u(x, t-r))$.

(c) (i) A non-negative weak solution u of problem (DS) is a function $u \in L^{\infty}(\Omega)$ satisfying

$$
\int_{\Omega} \phi(u) \Delta \zeta+f(u) h(u) \zeta d x=0
$$

for all $\zeta \in C^{2}(\bar{\Omega})$ with $\zeta \geq 0$ and $\zeta=0$ on $\partial \Omega$.

(ii) A non-negative classical solution $u$ of problem (DS) is a function $u \in C(\bar{\Omega})$ for which $\Delta \phi(u) \in C^{2}(\Omega)$ and satisfies (DS) pointwise.

In order to prove existence and uniqueness for the delay problem (D) we will first need the result for the non-delay problem $(\Pi)$, which is nonautonomous and inhomogeneous. We prove only that which is sufficient for our analysis of the delay problem.

Lemma 2.1. Let $H \in C(\bar{Q})$ and $0 \leq \eta \in C\left(\Gamma_{r}\right), \eta_{0}(x)=0$ in $\partial \Omega$. If (A1-A3) hold, then for all $T>0$ there exists a unique, non-negative weak solution $u$ of problem (П). Moreover, $u \in C\left(\overline{Q_{T}}\right)$. 
Proof. By [2, Theorem 2.1 and Theorem 2.3], there exists a $T>0$ such that (II) has a unique weak solution on $[0, T)$, and either $T=\infty$ or, $T<\infty$ with $\limsup _{t \rightarrow T^{-}}\|u(t)\|_{\infty}=\infty$. We show that $T=\infty$ via a standard comparison argument.

Let $e_{1}$ denote the principal eigenfunction of $-\Delta$ on $B$ with homogeneous Dirichlet boundary conditions, where $B$ is any open ball such that $\bar{\Omega} \subset B$. Let $\mu_{1}>0$ denote the corresponding principal eigenvalue and suppose $e_{1}$ is normalized such that $\left\|e_{1}\right\|_{\infty}=1$. Since $g(u) / u \rightarrow 0$ as $u \rightarrow \infty$ and $e_{1}>0$ on $\bar{\Omega}$, we can choose $c>0$ sufficiently large such that

$$
\|H\|_{L^{\infty}\left(Q_{T}\right)} g\left(c e_{1}(x)\right) \leq \mu_{1} c e_{1}(x)
$$

for all $x \in \bar{\Omega}$. Setting $w(x)=\phi^{-1}\left(c e_{1}(x)\right)$, we have

$$
\begin{aligned}
\partial_{t} w-\Delta \phi(w)-H(x, t) f(w) & \geq-\Delta\left(c e_{1}\right)-\|H\|_{L^{\infty}\left(Q_{T}\right)} g\left(c e_{1}\right) \\
& \geq-c\left(\Delta e_{1}+\mu_{1} e_{1}\right)=0
\end{aligned}
$$

in $Q_{T}$. Taking $c>0$ sufficiently large so that also $c e_{1} \geq \eta_{0}$ in $\bar{\Omega}$ ensures that $w$ is a classical supersolution of (П). It follows by comparison [2, Theorem $2.3]$ that $u \leq w$ almost everywhere in $\overline{Q_{T}}$ and so $u \in L^{\infty}\left(Q_{T}\right)$. Thus $T$ cannot be finite.

The continuity result is well-known [12, Theorem 6.1 and its Corollary].

Corollary 2.1. Let $0 \leq \eta \in C\left(\Gamma_{r}\right)$ and $\eta_{0}(x)=0$ for all $x \in \partial \Omega$. If $h \in C\left(\mathbb{R}^{+}\right)$and (A1-A3) hold, then for any $T>0$ there exists a unique non-negative weak solution $u$ of $(D)$. Moreover, $u$ is continuous on $\overline{Q_{T}}$ for all $T>0$. 
Proof. The method of proof mirrors that used for delay-differential equations [13] or delay reaction-diffusion equations [29].

Let $u_{1}$ be the unique, non-negative and continuous weak solution on $[0, r]$ of the problem

$$
\begin{aligned}
\partial_{t} u=\Delta \phi(u)+H_{1}(x, t) f(u) & \text { in } Q_{r}, \\
u=0 & \text { in } S_{r}, \\
u_{0}=\eta_{0} & \text { in } \bar{\Omega},
\end{aligned}
$$

where $H_{1}(x, t)=h(\eta(x, t-r))$ is continuous in $\bar{\Omega} \times[0, r]$. Such a solution is guaranteed to exist by Lemma 2.1. Clearly, $u_{1}$ is the unique weak solution of (D) on $[0, r]$. Now extend $u_{1}$ (but still denoting the extension by $u_{1}$ ) such that $u_{1}=\eta$ on $\Gamma_{r}$. Again by Lemma 2.1 there then exists a unique, non-negative and continuous weak solution $u_{2}$ on $[0,2 r]$ of the problem

$$
\begin{aligned}
\partial_{t} u=\Delta \phi(u)+H_{2}(x, t) f(u) & \text { in } Q_{2 r}, \\
u=0 & \text { in } S_{2 r}, \\
u_{0}=\eta_{0} & \text { in } \bar{\Omega},
\end{aligned}
$$

where $H_{2}(x, t)=h\left(u_{1}(x, t-r)\right)$ is continuous in $\bar{\Omega} \times[0,2 r]$. Clearly $u_{2}$ is then the unique weak solution of (D) on $[0,2 r]$. Continuing in this manner inductively we obtain a unique, non-negative and continuous weak solution $u_{j}$ of (D) on $[0, j r]$ for any positive integer $j$. For any $T>0$ we may choose $j$ such that $j r \geq T$ and obtain the unique, non-negative and continuous weak solution $u$ on $[0, T]$ as the restriction $u=\left.u_{j}\right|_{[0, T]}$, yielding the required result. 


\section{Some auxiliary degenerate elliptic and parabolic problems}

In proving the main convergence results for the delay problem (D) we will construct a contracting sequence of ordered upper and lower solutions of some related equations. We first require some additional assumptions, recalling that the function $g$ is given by equation (5).

(A4) $f$ is strictly increasing on $(0, \infty)$;

(A5) $G(x):=\int_{0}^{x} \frac{d s}{g(s)}$ exists for $x>0$ and $\lim _{x \rightarrow 0} G(x)=0$;

(A6) $g$ is strictly concave on $(0, \infty)$.

Remark 3.1. The following properties of $g$ follow easily from (A1-A6):

(g1) $g \in C^{1}(0, \infty) \cap C^{\alpha}\left(\mathbb{R}^{+}\right), g(0)=0$ and $g$ is strictly increasing;

(g2) $g(s) / s \rightarrow \infty$ as $s \rightarrow 0$;

(g3) $g(s) / s \rightarrow 0$ as $s \rightarrow \infty$.

Following [6], for $a \in C^{\alpha}(\bar{\Omega})$ we now consider the degenerate parabolic problem

$$
(P)\left\{\begin{aligned}
\partial_{t} z=\Delta \phi(z)+a(x) f(z) & \text { in } Q, \\
z=0 & \text { in } S_{\infty}, \\
z=z_{0} \geq 0 & \text { in } \bar{\Omega}
\end{aligned}\right.
$$

and its associated stationary problem

$$
(P S) \quad\left\{\begin{aligned}
\Delta \phi(z)+a(x) f(z)=0 & \text { in } \Omega, \\
z=0 & \text { in } \partial \Omega
\end{aligned}\right.
$$


where $a(x)$ may change sign in $\Omega$. Problems like $(\mathrm{P})$ and $(\mathrm{PS})$ have been studied by several authors, see $[5,20,21,22]$. The main interesting feature of $(\mathrm{PS})$ is the existence of non-negative, non-trivial solutions vanishing on open subsets of $\Omega$, so-called 'dead cores'. It can be shown that there exists a unique maximal solution of (DS) in the class of non-negative functions, but no such minimal solution exists and the situation is much more complicated than the case where $a(x)$ is positive. We describe the main features of the solutions of (P) and (PS) sufficient for our purposes.

The positivity set of $a$ is defined by

$$
\Omega^{+}(a)=\{x \in \Omega: a(x)>0\}
$$

and $\Omega_{i}^{+}(a)$ denote the connected components of $\Omega^{+}(a)$, for $i \in M$, where $M$ is an indexing set which is at most countable (due to the regularity of $\Omega$ and $a$ ). The stationary solution set $S(a)$ is defined to be the set of all non-negative weak solutions $u$ of (PS) satisfying $u>0$ on $\Omega^{+}(a)$.

We now observe that if $a>0$ on $\partial \Omega$, then $\partial \Omega \subset \overline{\bigcup_{i \in M} \Omega_{i}^{+}(a)}$ and so

$$
\overline{\Omega^{+}(a)}=\overline{\bigcup_{i \in M} \Omega_{i}^{+}(a)}=\overline{\bigcup_{i \in M} \Omega_{i}^{+}(a)} \cup \partial \Omega \subset \bigcup_{i \in M} \overline{\Omega_{i}^{+}(a)} \cup \partial \Omega .
$$

This allows us to apply [6, Theorem 2.1] (in the special case where $I=M$ and $S_{M}=N_{M}$, in the notation of that paper) to deduce that (PS) has at most one solution with the property of being positive on $\Omega^{+}(a)$. Then $[6$, Theorem 2.2(i)] guarantees the existence of such a solution (again taking $I=M)$. We summarize this and related results from [6] as follows:

Lemma 3.1. Let $a \in C^{\alpha}(\bar{\Omega})$ and suppose $\Omega^{+}(a)$ is non-empty. If (A1-A6) hold, then: 
(i) Maximality ([6, Theorems 2.1 and 2.2]): there exists $z^{*}=z^{*}(a) \in$ $C^{\alpha}(\bar{\Omega})$ such that $S(a)=\left\{z^{*}(a)\right\}$. Moreover, $z^{*}(a)$ is maximal with respect to the set of non-negative solutions of $(P S)$.

(ii) Monotonicity ([6, Lemma 2.4]): if $a_{1} \geq a_{2}$ in $\bar{\Omega}$ then $z^{*}\left(a_{1}\right) \geq z^{*}\left(a_{2}\right)$ in $\bar{\Omega}$.

(iii) Convergence ([6, Theorem 3.1] or [22, Theorem 8]): if $z_{0} \in L^{\infty}(\Omega)$ and $z_{0}>0$ on some open subset of $\Omega_{i}^{+}(a)$ for every $i \in M$, then the solution $z$ of $(P)$ converges to $z^{*}(a)$ in $L^{p}(\Omega)$, for any $p \geq 1$, as $t \rightarrow \infty$. If $n=1$, or $n \geq 2$ and $z$ is uniformly continuous on $\Omega \times[\varepsilon, \infty)$ for some $\varepsilon>0$, then convergence to $z^{*}(a)$ is in $L^{\infty}(\Omega)$.

Remark 3.2. The conditions on the initial data $z_{0}$ in Lemma 3.1 (iii) are clearly satisfied if $z_{0}$ is continuous and $z_{0}>0$ in $\Omega$ or, more generally, if $z(x, T)>0$ in $\Omega$ for some $T>0$ since problem $(P)$ is autonomous.

The proof of Lemma 3.1 can be found in the references given, but for exposition purposes we briefly outline the ideas here. Setting $v=\phi(z),(\mathrm{PS})$ is equivalent to

$$
\Delta v+a(x) g(v)=0 \quad \text { in } \Omega, \quad v=0 \quad \text { in } \partial \Omega .
$$

As in the proof of Lemma 2.1, (g3) guarantees the existence of supersolutions $\bar{v}(x)$ of (8) with arbitrarily large minimum over $\bar{\Omega}$. Similarly, (g2) ensures that arbitrarily small (weak) subsolutions $\underline{v}(x)$ of $(8)$ exist such that $\underline{v} \not \equiv 0$ on $\Omega_{i}^{+}(a)$, for every $i \in M$, again by using a principal eigenfunctions of $-\Delta$ on a small ball contained within the interior of each $\Omega_{i}^{+}(a)$. Each subsolution 
(respectively supersolution) then generates a monotonically increasing (respectively decreasing) solution $\underline{z}\left(t ; \underline{z}_{0}\right)$ of $(\mathrm{P})$ in time, (respectively $\bar{z}\left(t ; \bar{z}_{0}\right)$ ) where $\underline{z}_{0}(x)=\phi^{-1}(\underline{v}(x))$ (respectively $\left.\bar{z}_{0}(x)=\phi^{-1}(\bar{v}(x))\right)$. See $[11,22]$ for example. The limiting functions $\underline{z}^{*}(x)$ and $\bar{z}^{*}(x)$ are then weak solutions of (PS) satisfying $\bar{z}^{*} \geq \underline{z}^{*}>0$ on $\Omega^{+}(a)$ and the interval $\left[\underline{z}^{*}, \bar{z}^{*}\right]$ attracts (in $L^{p}(\Omega)$ ) all solutions of $(\mathrm{P})$ having initial data in $\left[\underline{z}^{*}, \bar{z}^{*}\right]$. The assumptions (A5-A6) ensure uniqueness via a maximum principle argument (see [6, Theorem 2.1$]$ ), so that $\underline{z}^{*}=\bar{z}^{*}=z^{*}(a)$, say. For any initial data $z_{0}$ satisfying (iii), $\underline{z}$ and $\bar{z}$ can then be chosen such that $z_{0} \in[\underline{z}, \bar{z}]$, so that $z\left(t ; z_{0}\right) \rightarrow\left[\underline{z}^{*}, \bar{z}^{*}\right]=\left\{z^{*}(a)\right\}$ in $L^{p}(\Omega)$ as $t \rightarrow \infty$.

Corollary 3.1. Let $z^{*}: C^{\alpha}(\bar{\Omega}) \rightarrow C^{\alpha}(\bar{\Omega}), a \mapsto z^{*}(a)$ be defined as in Lemma 3.1.

(a) Suppose $a^{(m)} \in C^{\alpha}(\bar{\Omega})$ is a monotonically increasing sequence such that $a^{(m)} \rightarrow a \in C^{\alpha}(\bar{\Omega})$ pointwise as $m \rightarrow \infty$. If $\Omega^{+}\left(a^{(m)}\right) \neq \emptyset$ for all $m \geq 1$, then $z^{*}\left(a^{(m)}\right) \rightarrow z^{*}(a)$ pointwise as $m \rightarrow \infty$.

(b) Suppose $b^{(m)} \in C^{\alpha}(\bar{\Omega})$ is a monotonically decreasing sequence such that $b^{(m)} \rightarrow b \in C^{\alpha}(\bar{\Omega})$ pointwise as $m \rightarrow \infty$. If $\Omega^{+}(b) \neq \emptyset$, then $z^{*}\left(b^{(m)}\right) \rightarrow$ $z^{*}(b)$ pointwise as $m \rightarrow \infty$.

Proof. (a). By Lemma $3.1 z^{*}\left(a^{(m)}\right)$ is an increasing sequence with $z^{*}\left(a^{(m)}\right) \leq$ $z^{*}(a)$. Hence $\left(z^{*}\left(a^{(m)}\right)\right)(x)$ converges pointwise for $x \in \bar{\Omega}$ as $m \rightarrow \infty$, with limit $v(x) \leq\left(z^{*}(a)\right)(x)$ say. Hence $\phi\left(z^{*}\left(a_{m}\right)(x)\right) \rightarrow \phi(v(x))$ pointwise for $x \in \bar{\Omega}$ as $m \rightarrow \infty$. Lebesgue's dominated convergence theorem then allows one to pass to the limit as $m \rightarrow \infty$ in the definition of weak solution for 
(PS), so that $v \in L^{\infty}(\Omega)$ satisfies

$$
\Delta \phi(v)+a(x) f(v)=0 \text { in } \Omega, \quad v=0 \text { in } \partial \Omega .
$$

By elliptic regularity it then follows that $v \in C^{\alpha}(\bar{\Omega})$ and $\phi(v) \in C^{2+\alpha}(\bar{\Omega})$.

It remains to show that $v=z^{*}(a)$. But for any $x \in \Omega^{+}(a)$ (i.e. $a(x)>$ $0)$ there exists $j$ such that $a^{(j)}(x)>0$, and so $x \in \Omega^{+}\left(a^{(j)}\right)$ and $0<$ $\left(z^{*}\left(a^{(j)}\right)\right)(x) \leq v(x)$. Since $x \in \Omega^{+}(a)$ was arbitrary we have $v>0$ in $\Omega^{+}(a)$ and so $v=z^{*}(a)$, by definition of $z^{*}$. This proves part (a).

Part (b) follows in an identical way, until one has to show $v=z^{*}(b)$. But for any $x \in \Omega^{+}(b)$ (i.e. $\left.b(x)>0\right),\left(z^{*}\left(b^{(m)}\right)\right)(x) \geq\left(z^{*}(b)\right)(x)>0$ and so (letting $m \rightarrow \infty), v(x) \geq\left(z^{*}(b)\right)(x)>0$. Since $x \in \Omega^{+}(b)$ was arbitrary we have $v>0$ in $\Omega^{+}(b)$ and so $v=z^{*}(b)$.

In the special case where $a$ is a positive constant, $a(x) \equiv k>0$ say, problem (PS) becomes

$$
\left(P S_{k}\right) \quad\left\{\begin{aligned}
\Delta \phi(z)+k f(z)=0 & \text { in } \Omega \\
z=0 & \text { in } \partial \Omega .
\end{aligned}\right.
$$

In what follows we denote the unique positive solution of $\left(P S_{k}\right)$ by $\psi_{k}$ (i.e. $\left.\psi_{k}=z^{*}(k)\right)$ and write $\Omega_{k}^{+}$for $\Omega^{+}\left(h \circ \psi_{k}\right)$, i.e. $\Omega_{k}^{+}=\left\{x \in \Omega: h\left(\psi_{k}(x)\right)>0\right\}$.

\section{Main results: global convergence for the delay problem}

The methods of this section are similar in spirit to those in $[5,11,21,22]$. The main difference however is that, unlike the non-delay cases considered in these references, solutions of (D) are not generally monotonic in time regardless of any specific choice of initial data (such as sub- and supersolutions of the associated elliptic problem). 
For $v, w \in C(\bar{\Omega})$ satisfying $v \leq w$ in $\bar{\Omega}$ we define the interval subset $[v, w]$ by

$$
[v, w]:=\{u \in C(\bar{\Omega}): v(x) \leq u(x) \leq w(x) \forall x \in \bar{\Omega}\} .
$$

We will say that $u(t) \rightarrow[v, w]$ in $C(\bar{\Omega})$ as $t \rightarrow \infty$ if

$$
v(x) \leq \liminf _{t \rightarrow \infty} u(x, t) \leq \limsup _{t \rightarrow \infty} u(x, t) \leq w(x)
$$

uniformly for $x \in \bar{\Omega}$.

We now make the following assumption concerning the delay function $h$ :

(A7) $h \in C^{\alpha}\left(\mathbb{R}^{+}\right) \cap C^{1}(0, \infty), h^{\prime}<0$ on $(0, \infty)$ and $k:=h(0)>0$.

It follows from (A7) and the boundary conditions on $\psi_{k}$ that $\Omega_{k}^{+} \neq \emptyset$.

Proposition 4.1. Suppose (A1-A7) hold and define the sequences $v_{n}$ and $w_{n}$ inductively by: $v_{1}=0, w_{1}=\psi_{k}, v_{n+1}=z^{*}\left(h\left(w_{n}\right)\right)$ and $w_{n+1}=z^{*}\left(h\left(v_{n+1}\right)\right)$. Then the sequences $v_{n}$ and $w_{n}$ are well-defined and have the following properties:

(i) $v_{n}, w_{n} \in C^{\alpha}(\bar{\Omega})$ for all $n \geq 1$ and $v_{2}>0$ in $\Omega_{k}^{+}$;

(ii) $v_{n} \leq w_{n}$ and $\left[v_{n+1}, w_{n+1}\right] \subset\left[v_{n}, w_{n}\right]$ for all $n \geq 1$;

(iii) $v_{n}, w_{n}$ converge pointwise to limits $v^{*}, w^{*} \in C^{\alpha}(\bar{\Omega})$, respectively, as $n \rightarrow \infty\left(\right.$ with $\left.v^{*} \leq w^{*}\right)$ and $v^{*}, w^{*}$ satisfy

$$
\begin{aligned}
\Delta \phi\left(v^{*}\right)+h\left(w^{*}\right) f\left(v^{*}\right)=0 & \text { in } \Omega, \\
\Delta \phi\left(w^{*}\right)+h\left(v^{*}\right) f\left(w^{*}\right)=0 & \text { in } \Omega, \\
v^{*}=w^{*}=0 & \text { in } \partial \Omega .
\end{aligned}
$$


Proof. One observes that since $\Omega^{+}\left(h\left(w_{1}\right)\right)=\Omega^{+}\left(h\left(\psi_{k}\right)\right)=\Omega_{k}^{+}$it follows that $v_{2}=z^{*}\left(h\left(w_{1}\right)\right)=z^{*}\left(h\left(\psi_{k}\right)\right)>0$ in $\Omega_{k}^{+}$.

Now, $v_{n+1}$ is defined to be the maximal solution of

$$
\Delta \phi(v)+h\left(w_{n}\right) f(v)=0 \quad \text { in } \Omega, \quad v=0 \quad \text { in } \partial \Omega
$$

and $w_{n+1}$ is defined to be the maximal solution of

$$
\Delta \phi(w)+h\left(v_{n+1}\right) f(w)=0 \quad \text { in } \Omega, \quad w=0 \quad \text { in } \partial \Omega .
$$

By the boundary conditions, $h\left(w_{n}(x)\right)=h\left(v_{n}(x)\right)=h(0)=k>0$ for any $x \in \partial \Omega$ and $n \geq 1$. Hence $\Omega^{+}\left(h\left(v_{n}\right)\right)$ and $\Omega^{+}\left(h\left(w_{n}\right)\right)$ are non-empty and so $z^{*}\left(h\left(w_{n}\right)\right)$ and $z^{*}\left(h\left(v_{n+1}\right)\right)$ are well defined. Elliptic regularity then yields part (i).

We show first by induction that $v_{n} \leq w_{n}$ for all $n \geq 1$. The case $n=1$ is trivially true. Suppose that $v_{n} \leq w_{n}$. Then

$$
0=\Delta \phi\left(v_{n+1}\right)+h\left(w_{n}\right) f\left(v_{n+1}\right) \leq \Delta \phi\left(v_{n+1}\right)+h\left(v_{n}\right) f\left(v_{n+1}\right),
$$

so that $v_{n+1}$ is a subsolution of the problem satisfied by $w_{n}$, namely,

$$
\Delta \phi\left(w_{n}\right)+h\left(v_{n}\right) f\left(w_{n}\right)=0 \quad \text { in } \Omega, \quad w_{n}=0 \quad \text { in } \partial \Omega .
$$

Hence $v_{n+1} \leq w_{n}$. But then

$$
0=\Delta \phi\left(v_{n+1}\right)+h\left(w_{n}\right) f\left(v_{n+1}\right) \leq \Delta \phi\left(v_{n+1}\right)+h\left(v_{n+1}\right) f\left(v_{n+1}\right),
$$

so that $v_{n+1}$ is a subsolution of $(10)$ and hence $v_{n+1} \leq w_{n+1}$ as required.

Next we show by induction that $\left[v_{n+1}, w_{n+1}\right] \subset\left[v_{n}, w_{n}\right]$ for all $n \geq 1$. For $n=1$ we trivially have $0=v_{1} \leq v_{2}$ and

$$
0=\Delta \phi\left(w_{2}\right)+h\left(v_{2}\right) f\left(w_{2}\right) \leq \Delta \phi\left(w_{2}\right)+k f\left(w_{2}\right),
$$


so that $w_{2}$ is a subsolution of problem $\left(P S_{k}\right)$, and hence $w_{2} \leq \psi_{k}=w_{1}$. Suppose now that $v_{n} \leq v_{n+1}$ and $w_{n+1} \leq w_{n}$. Then

$$
0=\Delta \phi\left(v_{n+1}\right)+h\left(w_{n}\right) f\left(v_{n+1}\right) \leq \Delta \phi\left(v_{n+1}\right)+h\left(w_{n+1}\right) f\left(v_{n+1}\right),
$$

so that $v_{n+1}$ is a subsolution of the problem

$$
\Delta \phi(v)+h\left(w_{n+1}\right) f(v)=0 \quad \text { in } \Omega, \quad v=0 \quad \text { in } \partial \Omega
$$

and hence $v_{n+1} \leq v_{n+2}$. Similarly,

$$
0=\Delta \phi\left(w_{n+2}\right)+h\left(v_{n+2}\right) f\left(w_{n+2}\right) \leq \Delta \phi\left(w_{n+2}\right)+h\left(v_{n+1}\right) f\left(w_{n+2}\right)
$$

so that $w_{n+2}$ is a subsolution of the problem

$$
\Delta \phi(w)+h\left(v_{n+1}\right) f(w)=0 \quad \text { in } \Omega, \quad w=0 \quad \text { in } \partial \Omega
$$

and hence $w_{n+2} \leq w_{n+1}$, as required. This completes part (ii) of the proof.

Since $v_{n}$ and $w_{n}$ are bounded monotonic sequences, the pointwise limits $v_{n}(x) \rightarrow v^{*}(x)$ and $w_{n}(x) \rightarrow w^{*}(x)$ exist for all $x \in \bar{\Omega}$ as $n \rightarrow \infty$, and $v^{*} \leq w^{*}$. By Lebesgue's monotone convergence theorem we may pass to the limit as $n \rightarrow \infty$ in the definition of weak solution, to obtain $v^{*}, w^{*} \in L^{\infty}(\Omega)$ as weak solutions of

$$
\begin{aligned}
\Delta \phi\left(v^{*}\right)+h\left(w^{*}\right) f\left(v^{*}\right)=0 & \text { in } \Omega, \\
\Delta \phi\left(w^{*}\right)+h\left(v^{*}\right) f\left(w^{*}\right)=0 & \text { in } \Omega, \\
v^{*}=w^{*}=0 & \text { in } \partial \Omega .
\end{aligned}
$$

Elliptic regularity then yields part (iii). 
Remark 4.1. Assumption (A1) and [18, Theorem 2.5] ensure that solutions of $(P)$ are in fact precompact in $C(\bar{\Omega})$. In this case the $L^{p}$-convergence referred to in Lemma 3.1 (iii) can be strengthened to convergence in $C(\bar{\Omega})$.

Proposition 4.2. Let $0 \leq \eta \in C\left(\Gamma_{r}\right)$ and $\eta_{0}(x)=0$ for all $x \in \partial \Omega$. Suppose that the solution $u$ of $(D)$ satisfies $u(x, t)>0$ in $\Omega \times[T, \infty)$, for some $T>r$ and let $v^{*}$ and $w^{*}$ be as in Proposition 4.1. If (A1-A7) hold, then $u(t) \rightarrow\left[v^{*}, w^{*}\right]$ in $C(\bar{\Omega})$ as $t \rightarrow \infty$.

Proof. We first prove that $u(t) \rightarrow\left[v_{n}, w_{n}\right]$ in $C(\bar{\Omega})$ as $t \rightarrow \infty$ for all $n \geq 1$, where $v_{n}, w_{n}$ are as in from Proposition 4.1.

By classical regularity theory for uniformly parabolic equations, the solution $u$ of (D) is a classical solution for $t>T$, and so from (A7) we have $\partial_{t} u-\Delta \phi(u)=h(u(x, t-r)) f(u) \leq k f(u)$ for $t>T$. Consequently, $u$ is a classical subsolution of Problem (P) with $a(x) \equiv k$ and $z(x, T)=u(x, T)$. Hence, by comparison, $u \leq \bar{z}_{1}$ where $\bar{z}_{1}$ is the solution of

$$
\begin{aligned}
\partial_{t} z=\Delta \phi(z)+k f(z) & \text { in } \Omega \times(T, \infty), \\
z=0 & \text { in } \partial \Omega \times(T, \infty), \\
z(x, T)=u(x, T) & \text { in } \bar{\Omega} .
\end{aligned}
$$

Since $\Omega^{+}(k)=\Omega$ and $z(x, T)>0$ in $\Omega$, it follows from Lemma 3.1 (iii) and Remark 4.1 that $\bar{z}_{1}(t) \rightarrow \psi_{k}$ in $C(\bar{\Omega})$ as $t \rightarrow \infty$. Hence $u(t) \rightarrow\left[0, w_{1}\right]$ in $C(\bar{\Omega})$ as $t \rightarrow \infty$.

We now proceed by induction. We have just shown that $u(t) \rightarrow\left[v_{1}, w_{1}\right]$ in $C(\bar{\Omega})$ as $t \rightarrow \infty$, where $v_{1}=0$ and $w_{1}=\psi_{k}$. If $u(t) \rightarrow\left[v_{n}, w_{n}\right]$ in $C(\bar{\Omega})$ as $t \rightarrow \infty$ then, for any $m \in \mathbb{N}$, there exists $\tau_{n}>T+r$ such that 
$u(x, t-r) \leq w_{n}(x)+1 / m$ for all $x \in \Omega$ and $t>\tau_{n}$. Hence for $t>\tau_{n}$ we have $\partial_{t} u-\Delta \phi(u) \geq h\left(w_{n}(x)+1 / m\right) f(u)$ and so $u$ is a supersolution of $(\mathrm{P})$ with $a(x)=a_{n}^{(m)}(x):=h\left(w_{n}(x)+1 / m\right)$ and $z\left(x, \tau_{n}\right)=u\left(x, \tau_{n}\right)>0$ in $\Omega$. Hence, $u \geq \underline{z}_{n}^{(m)}$, where $\underline{z}_{n}^{(m)}$ is the solution of

$$
\begin{aligned}
\partial_{t} z=\Delta \phi(z)+a_{n}^{(m)}(x) f(z) & \text { in } \Omega \times\left(\tau_{n}, \infty\right), \\
z=0 & \text { in } \partial \Omega \times\left[\tau_{n}, \infty\right), \\
z\left(x, \tau_{n}\right)=u\left(x, \tau_{n}\right)>0 & \text { in } \Omega .
\end{aligned}
$$

By Lemma 3.1 (iii) and Remark 4.1, $\bar{z}_{n}^{(m)}(t) \rightarrow z^{*}\left(a_{n}^{(m)}\right)$ in $C(\bar{\Omega})$ as $t \rightarrow \infty$. Hence

$$
\liminf _{t \rightarrow \infty} u(x, t) \geq z^{*}\left(a_{n}^{(m)}\right)
$$

uniformly in $x$ for all $m \geq 1$. Clearly, $a_{n}^{(m)}$ is monotonically increasing in $m$ and $a_{n}^{(m)} \rightarrow a_{n} \in C^{\alpha}(\bar{\Omega})$ as $m \rightarrow \infty$, where $a_{n}(x):=h\left(w_{n}(x)\right)$. For any $x \in$ $\partial \Omega, a_{n}^{(m)}(x)=h(1 / m)>0$ for $m$ sufficiently large, and so $\Omega^{+}\left(a_{n}^{(m)}\right)$ is nonempty for such $m$. By Corollary 3.1(a), $\lim _{m \rightarrow \infty} z^{*}\left(a_{n}^{(m)}(x)\right)=z^{*}\left(a_{n}(x)\right)=$ $z^{*}\left(h\left(w_{n}\right)\right)=v_{n+1}$. Thus,

$$
\liminf _{t \rightarrow \infty} u(x, t) \geq v_{n+1}
$$

uniformly in $x$.

Similarly it now follows that, for any $m \in \mathbb{N}$, there then exists $t_{n}>\tau_{n}$ such that $u(x, t-r) \geq\left[v_{n+1}(x)-1 / m\right]_{+}:=\max \left\{v_{n+1}(x)-1 / m, 0\right\}$ for all $x \in \bar{\Omega}$ and $t>t_{n}$. Hence for $t>t_{n}$ we have $\partial_{t} u-\Delta \phi(u) \leq h\left(v_{n+1}(x)-1 / m\right) f(u)$ and so $u$ is a subsolution of $(\mathrm{P})$ with $a(x)=b_{n}^{(m)}(x):=h\left(\left[v_{n+1}(x)-1 / m\right]_{+}\right)$ 
and $z\left(x, t_{n}\right)=u\left(x, t_{n}\right)>0$. Hence, $u \leq \bar{z}_{n}^{(m)}$, where $\bar{z}_{n}^{(m)}$ is the solution of

$$
\begin{aligned}
\partial_{t} z=\Delta \phi(z)+b_{n}^{(m)}(x) f(z) & \text { in } \Omega \times\left(t_{n}, \infty\right), \\
z=0 & \text { in } \partial \Omega \times\left[t_{n}, \infty\right), \\
z\left(x, t_{n}\right)=u\left(x, t_{n}\right)>0 & \text { in } \Omega .
\end{aligned}
$$

Again by Lemma 3.1 (iii) and Remark 4.1, $\bar{z}_{n}^{(m)}(t) \rightarrow z^{*}\left(b_{n}^{(m)}\right)$ in $C(\bar{\Omega})$ as $t \rightarrow \infty$. Hence

$$
\limsup _{t \rightarrow \infty} u(x, t) \leq z^{*}\left(b_{n}^{(m)}\right)
$$

uniformly in $x$ for all $m \geq 1$. Clearly, $b_{n}^{(m)}$ is monotonically decreasing in $m$ and $b_{n}^{(m)} \rightarrow b_{n} \in C^{\alpha}(\bar{\Omega})$ as $m \rightarrow \infty$, where $b_{n}(x):=h\left(v_{n+1}(x)\right)$. For any $x \in \partial \Omega, b_{n}(x)=h(0)=k>0$ and so $\Omega^{+}\left(b_{n}\right)$ is non-empty. By Corollary 3.1(b), $\lim _{m \rightarrow \infty} z^{*}\left(b_{n}^{(m)}(x)\right)=z^{*}\left(b_{n}(x)\right)=z^{*}\left(h\left(v_{n+1}\right)\right)=w_{n+1}$ and thus

$$
\limsup _{t \rightarrow \infty} u(x, t) \leq w_{n+1}
$$

uniformly in $x$. Hence $u(t) \rightarrow\left[v_{n+1}, w_{n+1}\right]$ in $C(\bar{\Omega})$ as $t \rightarrow \infty$ and so $u(t) \rightarrow$ $\left[v_{n}, w_{n}\right]$ in $C(\bar{\Omega})$ as $t \rightarrow \infty$ for all $n \geq 1$, by induction.

Finally, since $v_{n}$ and $w_{n}$ are bounded sequences in $C^{\alpha}(\bar{\Omega})$, there exist subsequences $v_{n_{j}}$ and $w_{n_{j}}$ such that $v_{n_{j}} \rightarrow v^{*}$ and $w_{n_{j}} \rightarrow w^{*}$ in $C(\bar{\Omega})$ as $j \rightarrow \infty$. Hence $u(t) \rightarrow\left[v^{*}, w^{*}\right]$ in $C(\bar{\Omega})$ as $t \rightarrow \infty$.

Remark 4.2. Eventual positivity of solutions of $(D)$ is by no means obvious in the general case and we do not pursue this interesting question here. See 
[8] for results in this direction in the non-delay case. However, under the assumptions of Theorem 4.1 below, such positivity is guaranteed. Positivity can also be ensured when the initial condition $\eta_{0}$ is assumed positive in $\Omega$. The reason is as follows. By Lipschitz continuity of $f$ and the boundedness of $u, u$ is a supersolution of the problem

$$
\begin{aligned}
\partial_{t} z=\Delta \phi(z)-M z & \text { in } Q_{T}, \\
z=0 & \text { in } S_{T}, \\
z(x, 0)=\eta_{0}(x)>0 & \text { in } \Omega
\end{aligned}
$$

for $M$ sufficiently large. By (A1) (in particular, using $u \phi^{\prime}(u)<\gamma \phi(u)$ ) one can then show that $\phi^{-1}\left(\epsilon \exp (-c t) e_{1}(x)\right)$ is a subsolution of (14) on any compact subset of $\Omega$, for $\epsilon>0$ sufficiently small and $c>0$ sufficiently large. It follows that $u \geq z>0$ in $Q_{T}$ for all $T>0$.

We are now in a position to establish the main result of this paper, that of global convergence of solutions of (D) to a unique positive equilibrium of (DS). This requires all of the assumptions (A1-A7), which we now summarize in a more convenient, but equivalent form.

$\left(H_{\phi}\right) \phi \in C^{1}\left(\mathbb{R}^{+}\right), \phi(0)=\phi^{\prime}(0)=0, \phi^{\prime}>0$ on $(0, \infty), \phi^{-1}$ exists and $\phi^{-1} \in C^{\alpha}\left(\mathbb{R}^{+}\right)$, there exist $\gamma, \delta>0$ such that $\phi$ is convex on $(0, \delta)$ and $u \phi^{\prime}(u)<\gamma \phi(u)$ on $(0, \infty)$

$\left(H_{f}\right) f \in C^{1}\left(\mathbb{R}^{+}\right), f(0)=0$ and $f$ is strictly increasing.

$\left(H_{h}\right) h \in C^{\alpha}\left(\mathbb{R}^{+}\right) \cap C^{1}(0, \infty), k:=h(0)>0$ and $h^{\prime}<0$ on $(0, \infty)$.

$\left(H_{g}\right) g$ is strictly concave on $(0, \infty), \lim _{s \rightarrow \infty} g(s) / s=0, G(x):=\int_{0}^{x} \frac{d s}{g(s)}$ exists and $\lim _{x \rightarrow 0} G(x)=0$. 
$\left(H_{\eta}\right) 0 \leq \eta \in C\left(\Gamma_{r}\right), \eta_{0}(x)=0$ in $\partial \Omega$ and $\eta_{0} \not \equiv 0$

Theorem 4.1. Let $\left(H_{\phi}\right),\left(H_{f}\right),\left(H_{h}\right),\left(H_{g}\right)$ and $\left(H_{\eta}\right)$ hold and let $\psi_{k}$ denote the unique positive solution of problem $\left(P S_{k}\right)$. Suppose also that

(A8) there exists $\beta>0$ such that $\frac{\phi(u) h(u)}{f(u)}$ is non-decreasing on $(0, \beta)$.

If $\left\|\psi_{k}\right\|_{\infty}<\beta$, then

(i) there exists a time $T_{0}=T_{0}(\eta) \geq 0$ such that the solution $u$ of the delay problem $(D)$ satisfies $u(x, t)>0$ in $\Omega \times\left[T_{0}, \infty\right)$;

(ii) there exists a unique non-negative, non-trivial solution $\varphi$ of problem $(D S)$ and $\varphi>0$ in $\Omega$;

(iii) the solution $u$ of the delay problem $(D)$ converges in $C(\bar{\Omega})$ to $\varphi$ as $t \rightarrow \infty$.

Proof. We first claim that $h(u)>0$ on $(0, \beta)$. For if not, then by $\left(H_{h}\right)$ there exists $q \in(0, \beta)$ such that $h(q)=0$ and $h^{\prime}(q)<0$. But then

$$
\begin{aligned}
(\phi h / f)^{\prime}(q) & =\left[f(q) h^{\prime}(q) \phi(q)+f(q) h(q) \phi^{\prime}(q)-f^{\prime}(q) h(q) \phi(q)\right] / f^{2}(q) \\
& =h^{\prime}(q) \phi(q) / f(q)<0
\end{aligned}
$$

contradicting the assumption that $\phi(u) h(u) / f(u)$ is non-decreasing on $(0, \beta)$.

We already know that $\limsup u(x, t) \leq \psi_{k}(x)$, uniformly in $x$. Hence, since $\psi_{k}<\beta$ in $\bar{\Omega}$, there exists $T_{1}$ such that $u(x, t)<\beta$ in $\bar{\Omega} \times\left[T_{1}, \infty\right)$. For $t \geq T_{1}+r, h(u(x, t-r))>0$ and so $u$ is a supersolution of the generalized 
porous medium-type equation

$$
\begin{aligned}
\partial_{t} z=\Delta \phi(z) & \text { in } \Omega \times\left(T_{1}+r, \infty\right), \\
z=0 & \text { in } \partial \Omega \times\left(T_{1}+r, \infty\right), \\
z\left(x, T_{1}+r\right)=u\left(x, T_{1}+r\right) & \text { in } \bar{\Omega} .
\end{aligned}
$$

From $\left(H_{\phi}\right)$, and in particular the convexity of $\phi$ near zero, the well-known positivity result $\left[9\right.$, Theorem 5] ensures the existence of a time $T_{0} \geq T_{1}+r$ such that the solution $z$ of $(15-17)$ satisfies $z>0$ in $\Omega \times\left[T_{0}, \infty\right)$. This yields the required positivity result (i). In particular this now allows us to make use of Proposition 4.2.

Since $\psi_{k}<\beta$ we also have that $\Omega_{k}^{+}=\Omega$ since $h\left(\psi_{k}\right)>0$ in $\Omega$. Hence $v^{*} \geq v_{2}>0$ in $\Omega$ by Proposition 4.1 (i). Multiplying (11) by $\phi\left(w^{*}\right)$, (12) by $\phi\left(v^{*}\right)$ and integrating, we obtain

$$
0=\int_{\Omega} f\left(v^{*}\right) f\left(w^{*}\right)\left[\frac{\phi\left(w^{*}\right) h\left(w^{*}\right)}{f\left(w^{*}\right)}-\frac{\phi\left(v^{*}\right) h\left(v^{*}\right)}{f\left(v^{*}\right)}\right] d x .
$$

Since $0<v^{*} \leq w^{*} \leq \psi_{k}<\beta$ in $\Omega$, it now follows from the monotonicity of $\phi h / f$ on $(0, \beta)$ that $v^{*}=w^{*}$. Consequently the solution $u$ of $(\mathrm{D})$ converges in $C(\bar{\Omega})$ to $v^{*}$ as $t \rightarrow \infty$, where $v^{*}$ is a positive solution of problem (DS). It remains only to show that $v^{*}$ is the unique, non-negative, non-trivial solution of (DS).

Setting $z=\phi(u)$, problem (DS) is equivalent to

$$
\Delta z+F(z)=0 \quad \text { in } \Omega, \quad z=0 \quad \text { in } \partial \Omega
$$

where $F(z):=h\left(\phi^{-1}(z)\right) g(z)$. By $\left(H_{h}\right)$ and $\left(H_{g}\right), \limsup F(z) / z \leq 0$ and $\lim _{z \rightarrow 0} F(z) / z=+\infty$. It follows from standard results for sublinear elliptic equations $[1,7]$ that there exists a maximal positive solution $\hat{z}$ of (19). Equivalently, there exists a maximal positive solution $\varphi:=\phi^{-1}(\hat{z}) \geq v^{*}>0$ of 
(DS). By $\left(H_{h}\right), \varphi$ is a subsolution of $\left(P S_{k}\right)$ and so $\varphi \leq \psi_{k}<\beta$ in $\Omega$. Now, for any non-negative non-trivial solution $U$ of (DS) we have $U \leq \varphi$ and so $h(U) \geq h(\varphi)>0$. Thus $U>0$ in $\Omega$ by the maximum principle. In the obvious way, from (DS) we also have

$$
0=\int_{\Omega} \phi(U) \phi(\varphi)\left[\frac{f(\varphi) h(\varphi)}{\phi(\varphi)}-\frac{f(U) h(U)}{\phi(U)}\right] d x .
$$

From $\left(H_{h}\right)$ and $(\mathrm{A} 8)$ we now have that

$$
\left[\frac{f(u) h(u)}{\phi(u)}\right]^{\prime}=\frac{2 h^{\prime}(u) f(u)}{\phi(u)}-\frac{f(u)^{2}}{\phi(u)^{2}}\left[\frac{\phi(u) h(u)}{f(u)}\right]^{\prime}<0
$$

on $(0, \beta)$ and so $f h / \phi$ is strictly decreasing on $(0, \beta)$. Hence, by $(20), U=\varphi$ and so $\varphi$ is the unique, non-negative, non-trivial solution of (DS), proving part (ii). In particular it follows that $v^{*}=\varphi$, proving part (iii).

Remark 4.3. The requirement that $\left\|\psi_{k}\right\|_{\infty}<\beta$ in Theorem 4.1 can obviously be weakened to $\left\|w^{*}\right\|_{\infty}<\beta$, where $w^{*}$ is as in Proposition 4.1. The choice of $\psi_{k}$ in the statement of the Theorem 4.1 has been made for the sake of explicitness and applicability, since it is more easily obtained via solution of $\left(P S_{k}\right)$ only.

\section{Some special cases and discussion}

In this final section we consider the power law nonlinearities $\phi(u)=u^{m}$ and $f(u)=u^{p}$ where $m>p \geq 1$ so that (D) becomes

$$
\partial_{t} u=\Delta u^{m}+u^{p} h(u(x, t-r)),
$$


with Dirichlet boundary conditions and initial data $\eta$ satisfying $\left(H_{\eta}\right)$. Hypotheses $\left(H_{\phi}\right),\left(H_{f}\right)$ and $\left(H_{g}\right)$ are easily seen to be true. It is also known (see [4]) that $\psi_{k}=k^{1 /(m-p)} \psi$, where $\psi$ is the unique positive solution of

$$
\Delta \psi^{m}+\psi^{p}=0 \quad \text { in } \Omega, \quad \psi=0 \quad \text { in } \partial \Omega .
$$

For suitable $h$, the hypotheses of Theorem 4.1 will then hold for all $k$ satisfying

$$
0<k<\left(\frac{\beta}{\|\psi\|_{\infty}}\right)^{m-p} .
$$

In the case of a one-dimensional domain $\Omega=(0, \pi)$ one easily obtains by quadrature that

$$
\|\psi\|_{\infty}=\left(\frac{(m+p) \pi^{2}}{8 m I^{2}}\right)^{\frac{1}{m-p}}, \quad I:=\int_{0}^{\pi / 2} \sin ^{\frac{m+p}{m-p}} \theta d \theta .
$$

We now consider some specific forms for $h$ satisfying $\left(H_{h}\right)$.

Example 5.1 (A generalized logistic). Consider the degenerate parabolic delay problem

$$
\partial_{t} u=\Delta u^{m}+k u^{p}\left(1-u^{q}(x, t-r)\right)
$$

with appropriate boundary and initial conditions, $k>0$ and $q>0$. Here, $h(s)=k\left(1-s^{q}\right)$ clearly satisfies $\left(H_{h}\right)$. Now, $\phi(u) h(u) / f(u)=k u^{m-p}\left(1-u^{q}\right)$ is non-decreasing for all $u \in(0, \beta)$, where $\beta=[(m-p) /(m-p+q)]^{1 / q}$. From (21) the hypotheses of Theorem 4.1 will be satisfied provided that

$$
k<k_{0}(m, p, q):=\left(\frac{m-p}{(m-p+q)\|\psi\|_{\infty}^{q}}\right)^{(m-p) / q} .
$$

Hence for $k<k_{0}$ solutions of (D) converge to the unique positive solution of

$$
\Delta \varphi^{m}+k \varphi^{p}\left(1-\varphi^{q}\right)=0 \quad \text { in } \Omega, \quad \varphi=0 \quad \text { in } \partial \Omega
$$

as $t \rightarrow \infty$. 
Example 5.2 (The logistic case). For $p=q=1$ we have the logistic term considered in [10], but here with nonlinear degenerate diffusion $\phi(u)=u^{m}$ for $m>1$. Hence we have global convergence to $\varphi$ as $t \rightarrow \infty$ if

$$
k<k_{0}(m, 1,1)=\left(\frac{1-\frac{1}{m}}{\|\psi\|_{\infty}}\right)^{(m-1)} .
$$

where $\varphi$ is the unique positive solution of

$$
\Delta \varphi^{m}+k \varphi(1-\varphi)=0 \quad \text { in } \Omega, \quad \varphi=0 \quad \text { in } \partial \Omega .
$$

When $\Omega=(0, \pi)$,

$$
k_{0}(m, 1,1)=\left(\frac{8 m I^{2}}{(m+1) \pi^{2}}\right)\left(1-\frac{1}{m}\right)^{m-1} .
$$

One observes that $k_{0}(m, 1,1) \rightarrow 0$ as $m \rightarrow 1^{+}$and $k_{0}(m, 1,1) \rightarrow 8 /\left(e \pi^{2}\right)$ as $m \rightarrow \infty$.

Example 5.2 shows that the destabilization caused by the Hopf bifurcation (as $r$ increases) of small positive solutions in [10] for the case $m=1$, does not occur in the degenerate diffusion case $m>1$. The reason is as follows. For $m>1$, it is known (see [11]) that the unique positive equilibrium of (23), $\varphi=\varphi_{k}$, is increasing in $k$ and $\left\|\varphi_{k}\right\|_{\infty} \rightarrow 0$ as $k \rightarrow 0^{+}$. Thus for $m>1$, small positive solutions corresponds to small $k$. But since $k_{0}$ is independent of $r$, all non-trivial solutions converge to the equilibrium $\varphi_{k}$ as $t \rightarrow \infty$, for all $k \in\left(0, k_{0}\right)$, regardless of the size of the delay $r$.

The question of convergence to equilibrium for values of $k$ larger than $k_{0}$ in the logistic problem remains open. The restriction on $k$ in Theorem 4.1 is imposed in order to guarantee uniqueness of solutions $\left(v^{*}, w^{*}\right)$ of $(11-13)$ satisfying $0<v^{*} \leq w^{*} \leq \psi_{k}$. The author has been unable to determine 
whether such uniqueness holds for all $k>0$ and is unaware of any results in the literature that guarantee uniqueness for such $k$. The only other results the author is aware of appear in [25, Proposition 5.4, Corollary 5.6], and are equivalent to requiring that

$$
k<\frac{\left(1-\frac{1}{m}\right)^{m-1}}{\left\|e_{0}\right\|_{\infty}}
$$

where $e_{0}$ is the unique positive solution of $-\Delta e_{0}=1$ in $\Omega$ with zero Dirichlet boundary conditions. For the case where $\Omega=(0, \pi)$ the bound in $(25)$ becomes $k<8\left(1-\frac{1}{m}\right)^{m-1} / \pi^{2}$; compare with (24). However, one would still need to check whether the condition in (25) is sufficient to guarantee that the attracting interval $\left[v^{*}, w^{*}\right]$ has $v^{*}>0$ in $\Omega$, as opposed to $v^{*}$ merely being non-negative and non-trivial (called semi-coexistence states in [25]).

Example 5.3. In this example we demonstrate that condition (A8) may hold with $\beta=\infty$ and therefore can be sufficient for global convergence of solutions of (D) for all $k>0$. We consider the problem

$$
\partial_{t} u=\Delta u^{m}+\frac{k u^{p}}{c+u^{q}(x, t-r)}
$$

for $c, q>0$ with Dirichlet boundary conditions. Here, $h(s)=k /\left(c+s^{q}\right)$ for $s \geq 0$. If $m \geq p+q$ then $\phi(u) h(u) / f(u)=k u^{m-p} /\left(c+u^{q}\right)$ is increasing on $(0, \infty)$ and so we may take $\beta=\infty$ in Theorem 4.1 to conclude that $\varphi$ is globally attractive for all $k>0$, where $\varphi$ is the unique positive solution of

$$
\Delta \varphi^{m}+\frac{k \varphi^{p}}{c+\varphi^{q}}=0 \quad \text { in } \Omega, \quad \varphi=0 \quad \text { in } \partial \Omega .
$$

If $m \in(p, p+q)$ however, then $\phi(u) h(u) / f(u)$ is only increasing on $(0, \beta)$, where

$$
\beta=\left(\frac{c(m-p)}{p+q-m}\right)^{1 / q}
$$


Thus, in the latter case, we can only ensure global attractivity of $\varphi$ for nonnegative solutions of (D) if

$$
k<k_{0}(m, p, q, c):=\left(\frac{c(m-p)}{(p+1-m)\|\psi\|_{\infty}^{q}}\right)^{(m-p) / q} .
$$

\section{References}

[1] H. Amann. On the existence of positive solution of nonlinear elliptic boundary value problems. Indiana Univ. Math. J., 21:125-146, 1971.

[2] J.R. Anderson. Local existence and uniqueness of solutions of degenerate parabolic equations. Comm. Partial Differential Equations, 16(1):105143, 1991.

[3] D. Aronson, M. G. Crandall, and L. A. Peletier. Stabilization of solutions of a degenerate nonlinear diffusion problem. Nonlinear Anal. TMA, 6:1001-1022, 1982.

[4] D. Aronson and L. A. Peletier. Large time behaviour of solutions of the porous medium equation in bounded domains. J. Differential Equations, 39:378-412, 1981.

[5] M. Badii, J.I. Diaz, and A. Tesei. Existence and attractivity results for a class of degenerate functional parabolic problems. Rend. Sem. Mar. Univ. Padova, 78:109-124, 1987.

[6] C. Bandle, M.A. Pozio, and A. Tesei. The asymptotic behaviour of the solutions of degenerate parabolic equations. Trans. Amer. Math. Soc., 303(2):487-501, 1987. 
[7] H. Berestycki and P. L. Lions. Some applications of the method of sub and supersolutions. In Bifurcation and Nonlinear Eigenvalue Problems, number 782 in Springer Lecture Notes, pages 16-41. Springer, 1980.

[8] M. Bertsch, R. Kersner, and L. A. Peletier. Positivity versus localization in degenerate diffusion equations. Nonlinear Anal. TMA, 9(9):987-1008, 1985 .

[9] M. Bertsch and L. A. Peletier. A positivity property of solutions of nonlinear diffusion equations. J. Differential Equations, 53:30-47, 1984.

[10] S. Busenberg and W. Huang. Stability and hopf bifurcation for a population delay model with diffusion effects. J. Differential Equations, 124:80-107, 1996.

[11] P. DeMottoni, A. Schiaffino, and A. Tesei. Attractivity properties of non-negative solutions for a class of nonlinear degenerate parabolic equations. Ann. Mat. Pura Appl., 136:35-48, 1984.

[12] E. DiBenedetto. Continuity of weak solutions to a general porous medium equation. Indiana Univ. Math. J., 32:83-118, 1983.

[13] J. K. Hale. Theory of Functional Differential Equations. Applied Mathematical Sciences 3. Springer-Verlag, New York, 1977.

[14] P. Hess, M.A. Pozio, and A. Tesei. Time-periodic solutions for a class of degenerate parabolic problems. Houston J. Math., 21(2):367-394, 1995.

[15] Y. Kuang and H.L. Smith. Convergence in lotka-volterra-type delay 
systems without instantaneous feedbacks. Proc. Roy. Soc. Edinburgh: Section A, 123(1):45-58, 1993.

[16] Y. Kuang and H.L. Smith. Convergence in lotka-volterra type diffusive delay systems without dominating instantaneous negative feedbacks. $J$. Australian Math. Soc.: Series B, 34(4):471-494, 1993.

[17] R. Laister. Global asymptotic behaviour in some functional parabolic equations. Nonlinear Anal. TMA, 50(3):347-361, 2002.

[18] M. Langlais and D. Phillips. Stabilization of solutions of nonlinear and degenerate evolution equations. Nonlinear Anal. TMA, 9(4):321-333, 1985.

[19] R.H. Martin and H.L. Smith. Reaction-diffusion systems with time delays - monotonicity, invariance, comparison and convergence. J. Reine Angew. Math., 413:1-35, 1991.

[20] M.Delgado and A. Suarez. Stability and uniqueness for cooperative degenerate lotka-volterra model. Nonlinear Anal. TMA, 49(6):757-778, 2002.

[21] M.A. Pozio and A. Tesei. Degenerate parabolic problems in population dynamics. Japan J. Appl. Math., 2:351-380, 1985.

[22] M.A. Pozio and A. Tesei. Support properties of solutions for a class of degenerate parabolic problems. Comm. Partial Differential Equations, 12:47-75, 1987. 
[23] A.A. Samarskii, V.A. Galaktionov, S.P. Kurdyumov, and A.P. Mikhailov. Blow-up in Quasilinear Parabolic Equations. Walter de Gruyter, Berlin, 1995.

[24] H.L. Smith. Monotone Dynamical Systems: An Introduction to the Theory of Competitive and Cooperative Systems. Mathematical Surveys and Monographs 41. American Mathematical Society, 2008.

[25] A. Suarez. Nonnegative solutions for a heterogeneous degenerate competition model. ANZIAM Journal, 46(2):273-297, 2004.

[26] J. Sun, B. Wu, and D. Zhang. Asymptotic behavior of solutions of a periodic diffusion equation. J. Inequal. Appl., 2010.

[27] J.L. Vazquez. The Porous Medium Equation: Mathematical Theory. Oxford Mathematical Monographs. Oxford University Press, USA, 2006.

[28] Y. Wang and J. Yin. Periodic solutions of a class of degenerate parabolic system with delays. J. Math. Anal. Appl., 380(1):57-68, 2011.

[29] J. Wu. Theory and Applications of Partial Functional Differential Equations. Applied Mathematical Sciences 119. Springer-Verlag, New York, 1996.

[30] Y. Yang, R. Zhang, C. Jin, and J. Yin. Existence of time periodic solutions for the nicholson's blowflies model with newtonian diffusion. Math. Meth. Appl. Sci., 33(7):922-934, 2010. 